\title{
Robust Consensus for Uncertain Multi-Agent Systems with Discrete-Time Dynamics
}

\author{
Dongkun Han*† and Graziano Chesi ${ }^{\ddagger}$ \\ Department of Electrical and Electronic Engineering, The University of Hong Kong, Pokfulam, Hong Kong
}

\begin{abstract}
SUMMARY
This paper investigates robust consensus for multi-agent systems with discrete-time dynamics affected by uncertainty. In particular, the paper considers multi-agent systems with single and double integrators, where the weighted adjacency matrix is a polynomial function of uncertain parameters constrained into a semialgebraic set. Firstly, necessary and sufficient conditions are provided for robust consensus based on the existence of a Lyapunov function polynomially dependent on the uncertainty. In particular, an upper bound on the degree required for achieving necessity is provided. Secondly, a necessary and sufficient condition is provided for robust consensus with single integrator and nonnegative weighted adjacency matrices based on the zeros of a polynomial. Lastly, it is shown how these conditions can be investigated through convex programming by exploiting linear matrix inequalities and sums of squares of polynomials. Some numerical examples illustrate the proposed results. Copyright (c) 2010 John Wiley \& Sons, Ltd.
\end{abstract}

Received ...

KEY WORDS: Multi-agent system; Discrete-time; Uncertainty; Robust consensus; Convex optimization.

\section{INTRODUCTION}

In recent years the consensus problems of multi-agent systems (or networked cooperative systems) are of great academic vitality, mainly owning to its wide applications in various areas including biological synchronization, formation control, sensor network and cooperative tracking control [1-4]. It has been well studied that the interaction structure of multi-agent systems is a deciding factor on whether the consensus can be achieved $[5,6]$. Specifically, to guarantee the consensability

\footnotetext{
†E-mail: dkhan@eee.hku.hk

${ }^{\ddagger}$ E-mail: chesi@eee.hku.hk

Copyright (c) 2010 John Wiley \& Sons, Ltd.

Prepared using rncauth.cls [Version: 2010/03/27 v2.00]
} 
requires that the communication graph contains a spanning tree for a fixed topological structure, while for a switching network, the union of communication graph has to obtain a spanning tree frequently enough as the system evolves [7-9].

In addition to the switching topology, many efforts have been made to construct a more realistic model of multi-agent systems. Various assumptions have been brought in such as unmeasurable inputs, misbehaving agents, unexpected disturbances on states and uncertain perturbations in communication $[6,10]$. This has led to the introduction of robust consensus, i.e. consensus achieved for all admissible uncertainties. In particular, [11] investigates robust consensus of multi-agent systems with input delays assuming the presence of asymmetric linear perturbations in the coupling weights of the communication network. [12] supposes that the control input affects the velocity states and depends linearly on uncertain parameters. [13] proposes a robust consensus controller and employs the proximity graph to model the communication constraints.

This paper investigates robust consensus for multi-agent systems with discrete-time dynamics affected by uncertainty. In particular, the paper considers multi-agent systems with single and double integrators, where the weighted adjacency matrix is a polynomial function of uncertain parameters constrained into a semialgebraic set. Firstly, necessary and sufficient conditions are provided for robust consensus based on the existence of a Lyapunov function polynomially dependent on the uncertainty. In particular, an upper bound on the degree required for achieving necessity is provided. Secondly, a necessary and sufficient condition is provided for robust consensus with single integrator and positive weighted adjacency matrices based on the zeros of a polynomial. Lastly, it is shown how these conditions can be investigated through convex programming by exploiting linear matrix inequalities (LMIs) and sums of squares of polynomials (SOS) (see e.g. [14] and references therein about SOS polynomials). Some numerical examples illustrate the proposed results.

It is worth remarking that existing works have not addressed yet the problems considered in this paper. Indeed, robust consensus for multi-agent systems with discrete-time dynamics has been considered only in the case of weighted adjacency matrices linearly affected by perturbations with some specific structures, see e.g. [11,12]. A preliminary version of this paper appeared in [15], which is extended in this paper by considering not multi-agent systems with single integrator but also with double integrator, and by providing an upper bound on the degree of the Lyapunov function required for achieving necessity in the conditions for robust consensus.

The paper is organized as follows. Section 2 introduces the problem formulation and provides some preliminaries about graph theory and SOS polynomials. Section 3 provides the proposed conditions for robust consensus are provided. Section 4 illustrates the proposed conditions with some numerical examples. Lastly, Section 5 concludes the paper with some final remarks. 


\section{PRELIMINARIES}

\subsection{Problem Formulation}

Notations: $\mathbb{N}, \mathbb{R}, \mathbb{C}$ : spaces of natural, real, and complex numbers; $A^{\prime}$ : transpose of $A ; A>0(A \geq$ $0)$ : symmetric positive definite (semidefinite) matrix $A ; 0_{n}$ : origin of $\mathbb{R}^{n} ; 1_{n}=(1, \ldots, 1)^{\prime} \mathbb{R}^{n} ; I_{n}$ : $n \times n$ identity matrix; $\operatorname{img}(A)$ : image of $A ; \operatorname{ker}(A)$ : null space of $A ; A \otimes B$ : Kronecker product of $A$ and $B ; \operatorname{adj}(A):$ adjoint of $A ; \operatorname{LCM}\{a, b, \ldots\}:$ least common multiplier of $a, b, \ldots$.

In graph theory, a weighted and directed graph $\mathscr{G}$ is defined as $\mathscr{G}=(\mathscr{A}, \mathscr{E}, G)$, where $\mathscr{A}=$ $\left\{A_{1}, \ldots, A_{n}\right\}$ describes the set of $n$ nodes of a multi-agent system, $\mathscr{E}$ is the set of directed edges belonging to $\mathscr{A} \times \mathscr{A}$ and $G$ is the weighted adjacency matrix. If the $(i, j)$-th entry of $G$ satisfies $G_{i j} \neq 0$, then there is a directed edge from the $j$-th node to the $i$-th node in $\mathscr{G}$. A directed path from $A_{i}$ to $A_{j}$ is defined as a sequence of directed edges $\left(A_{i}, A_{i 1}\right),\left(A_{i 1}, A_{i 2}\right), \ldots,\left(A_{i l}, A_{j}\right)$ in a directed network with distinct nodes $A_{i k}, k=1, \ldots, l$. If a node $i$ has the property that, for any node $j$ different from $i$, there is a directed path from $i$ to $j$, then the node $i$ is said to be a root. A directed tree is a directed graph where exactly one root exists and, except the root, every node in $\mathscr{G}$ has exactly one parent. If the edges of a directed tree connect all the nodes of the graph, we call this kind of directed tree as a spanning tree.

In this paper we investigate robust consensus for multi-agent systems with discrete-time dynamics affected by uncertainty, which can reflect the presence of partially or totally unknown control gains. In particular, for multi-agent systems with single integrator we consider the model

$$
x_{i}(k+1)=\frac{1}{\sum_{j=1}^{n} G_{i j}(\theta)} \sum_{j=1}^{n} G_{i j}(\theta) x_{j}(k), i=1, \ldots, n
$$

where $x_{i} \in \mathbb{R}$ is the state of the $i$-th node, $\theta \in \mathbb{R}^{r}$ is the vector of uncertain parameters, and $G$ : $\mathbb{R}^{r} \rightarrow \mathbb{R}^{n \times n}$ is a polynomial function. The vector of uncertain parameters is constrained according to

$$
\theta \in \Omega
$$

where $\Omega$ is the semialgebraic set defined by

$$
\Omega=\left\{\theta \in \mathbb{R}^{r}: s_{i}(\theta) \geq 0 \forall i=1, \ldots, h\right\}
$$

for some polynomials $s_{1}, \ldots, s_{h}: \mathbb{R}^{r} \rightarrow \mathbb{R}$. The system (1) can be rewritten in compact form as

$$
x(k+1)=D(\theta) x(k)
$$


where $D: \mathbb{R}^{r} \rightarrow \mathbb{R}^{n \times n}$ is defined by

$$
D_{i j}(\theta)=\frac{G_{i j}(\theta)}{\sum_{k=1}^{n} G_{i k}(\theta)}, i, j=1, \ldots, n
$$

Problem 1. To establish whether (1) achieves robust consensus, i.e.

$$
\lim _{k \rightarrow \infty} x_{i}(k)-x_{j}(k)=0 \forall i, j=1, \ldots, n \forall x(0) \in \mathbb{R}^{n} \forall \theta \in \Omega
$$

For multi-agent systems with double integrator, we consider the model

$$
\left\{\begin{aligned}
x_{i}(k+1) & =x_{i}(k)+\varrho_{i}(k) \\
\varrho_{i}(k+1) & =\varrho_{i}(k)+u_{i}(k)
\end{aligned}\right.
$$

with

$$
u_{i}(k)=k_{1} \sum_{j=1}^{n} G_{i j}(\theta)\left(x_{j}(k)-x_{i}(k)\right)+k_{2} \sum_{j=1}^{n} G_{i j}(\theta)\left(\varrho_{j}(k)-\varrho_{i}(k)\right)
$$

where $k_{1}, k_{2} \in \mathbb{R}$ are positive scalars representing coupling strengthes, and $x_{i}, \varrho_{i} \in \mathbb{R}$ denote the position and velocity states of the $i$-th agent. The system (7) can be rewritten in compact form as

$$
\left(\begin{array}{c}
x(k+1) \\
\varrho(k+1)
\end{array}\right)=\Gamma(\theta)\left(\begin{array}{c}
x(k) \\
\varrho(k)
\end{array}\right)
$$

where

$$
\Gamma(\theta)=\left(\begin{array}{cc}
I_{n} & I_{n} \\
-k_{1} L(\theta) & I_{n}-k_{2} L(\theta)
\end{array}\right)
$$

and $L(\theta) \in \mathbb{R}^{n \times n}$ is the uncertain Laplacian matrix given by

$$
\begin{aligned}
& L_{i j}(\theta)=-G_{i j}(\theta) \forall i \neq j \\
& L_{i i}(\theta)=-\sum_{j=1, j \neq i}^{n} L_{i j}(\theta) .
\end{aligned}
$$

Problem 2. To establish if (7) achieves robust consensus, i.e.

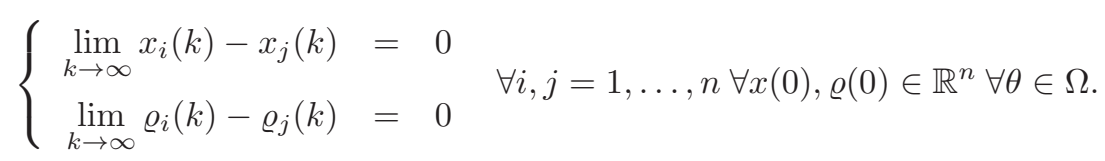

In the sequel we will assume that $G(\theta)$ is well-posed over $\Omega$, i.e.

$$
\sum_{k=1}^{n} G_{i k}(\theta) \neq 0 \quad \forall i=1, \ldots, n \forall \theta \in \Omega .
$$


Also, we will say that $G(\theta)$ is nonnegative if $G_{i j}(\theta) \geq 0$ for all $i, j=1, \ldots, n$ and for all $\theta \in \Omega$.

It is also useful to introduce the definition of (row) stochastic matrix, which is a nonnegative matrix with the property that all its row sums are 1 [16]. Let us observe that $D(\theta)$ is a stochastic matrix if $G(\theta)$ is nonnegative.

\subsection{SOS Polynomials}

A sufficient condition for establishing whether a polynomial is nonnegative consists of establishing if the polynomial is SOS, and this latter condition amounts to solving an LMI feasibility test as explained for instance in [14].

Specifically, let $f(\theta)$ be a polynomial of degree not greater than $2 m$ in $\theta \in \mathbb{R}^{r}$. Then, $f(\theta)$ can be written as

$$
f(\theta)=\theta^{\{m\}^{\prime}}(F+C(\delta)) \theta^{\{m\}}
$$

where $\theta^{\{m\}} \in \mathbb{R}^{\sigma(r, m)}$ is a vector containing all monomials of degree not greater than $m$ in $\theta$, whose number is given by

$$
\sigma(r, m)=\frac{(r+m) !}{r ! m !}
$$

$F$ is a symmetric matrix, and $C(\delta)$ is a linear parametrization of the linear subspace

$$
\mathcal{C}=\left\{C=C^{\prime}: \theta^{\{m\}^{\prime}} C \theta^{\{m\}}=0\right\} .
$$

The representation (14) is known as Gram matrix method and as square matrix representation (SMR). In [17] this representation was introduced to establish whether a polynomial is SOS through an LMI feasibility test. Indeed, $f(\theta)$ is SOS if there exist polynomials $f_{1}(\theta), f_{2}(\theta), \ldots$ such that

$$
f(\theta)=\sum_{i} f_{i}(\theta)^{2}
$$

and this condition holds if and only if there exists $\delta$ such that the following LMI holds:

$$
F+C(\delta) \geq 0
$$

This technique can also be used in the case of matrix polynomials as proposed in [18]. Specifically, let $M(\theta) \in \mathbb{R}^{s \times s}$ be a symmetric matrix polynomial of degree not greater than $2 m$ in $\theta \in \mathbb{R}^{r}$. Then, $M(\theta)$ can be written as

$$
M(\theta)=\Delta(\bar{M}+N(\delta), m, s)
$$

where

$$
\Delta(\bar{M}+N(\delta), m, s)=\left(\theta^{\{m\}} \otimes I_{s}\right)^{\prime}(\bar{M}+N(\delta))\left(\theta^{\{m\}} \otimes I_{s}\right),
$$


$\bar{M}$ is a symmetric matrix, and $N(\delta)$ is a linear parametrization of the linear subspace

$$
\mathcal{N}=\left\{N=N^{\prime}:\left(\theta^{\{m\}} \otimes I_{s}\right)^{\prime} N\left(\theta^{\{m\}} \otimes I_{s}\right)=0\right\} .
$$

The matrix polynomial $M(\theta)$ is said SOS if there exist matrix polynomials $M_{1}(\theta), M_{2}(\theta), \ldots$ such that

$$
M(\theta)=\sum_{i} M_{i}(\theta)^{\prime} M_{i}(\theta)
$$

and this condition holds if and only if there exists $\delta$ satisfying the LMI

$$
\bar{M}+N(\delta) \geq 0 .
$$

\section{CONDITIONS FOR ROBUST CONSENSUS}

\subsection{Single Integrator}

Let us introduce the polynomial

$$
\zeta(\theta)=\operatorname{LCM}\left\{\sum_{j=1}^{n} G_{i j}(\theta), i=1, \ldots, n\right\} .
$$

and let us define

$$
F(\theta)=\zeta(\theta) D(\theta)
$$

Let $V_{1} \in \mathbb{R}^{n \times(n-1)}$ be such that

$$
\left\{\begin{aligned}
\operatorname{img}\left(V_{1}\right) & =\operatorname{ker}\left(1_{n}^{\prime}\right) \\
V_{1}^{\prime} V_{1} & =I_{n-1}
\end{aligned}\right.
$$

and let us define

$$
D_{1}(\theta)=V_{1}^{\prime} D(\theta) V_{1}
$$

and

$$
F_{1}(\theta)=V_{1}^{\prime} F(\theta) V_{1} .
$$

The following result provides a sufficient and necessary condition for establishing whether (1) achieves robust consensus.

\section{Theorem 1}

Let $\tau$ be the degree of $G(\theta)$, and define

$$
\mu_{1}=n\left(n^{2}-n-2\right) \tau .
$$


The system (1) achieves robust consensus if and only if there exists a symmetric matrix polynomial $P(\theta) \in \mathbb{R}^{(n-1) \times(n-1)}$ of degree $d \leq \mu_{1}$ such that

$$
\left\{\begin{array}{l}
P(\theta)>0 \\
\zeta(\theta)^{2} P(\theta)-F_{1}(\theta)^{\prime} P(\theta) F_{1}(\theta)>0
\end{array} \quad \forall \theta \in \Omega .\right.
$$

Proof (Sufficiency) Suppose that (27) holds. From Lyapunov stability theorem for discrete-time linear systems, this implies that

$$
\left|\lambda_{i}\left(F_{1}(\theta)\right)\right|<|\zeta(\theta)| \quad \forall i=1, \ldots, n-1 \forall \theta \in \Omega
$$

where $\lambda_{i}\left(F_{1}(\theta)\right)$ is the $i$-th eigenvalue of $F_{1}(\theta)$. Since

$$
F_{1}(\theta)=\zeta(\theta) D_{1}(\theta)
$$

it hence follows that $D_{1}(\theta)$ is Schur for all $\theta \in \Omega$, i.e.

$$
\left|\lambda_{i}\left(D_{1}(\theta)\right)\right|<1 \forall i=1, \ldots, n-1 \forall \theta \in \Omega
$$

Since 1 is an eigenvalue of $D(\theta)$, we can denote the characteristic polynomial of $D(\theta)$ as

$$
\operatorname{det}(\lambda I-D(\theta))=(\lambda-1) \xi(\lambda, \theta)
$$

Since $1_{n}$ is an eigenvector of $D(\theta)$ corresponding to the eigenvalue 1 , it follows that the characteristic polynomial of $D_{1}(\theta)$ is given by

$$
\operatorname{det}\left(\lambda I-D_{1}(\theta)\right)=\xi(\lambda, \theta)
$$

i.e. $D_{1}(\theta)$ has the same eigenvalues of $D(\theta)$ except that the algebraic multiplicity of the eigenvalue 1 has been decreased of one. Hence, since $D_{1}(\theta)$ is Schur for all $\theta \in \Omega$, it follows that $D(\theta)$ has exactly one simple eigenvalue 1 and all the other eigenvalues with magnitude smaller than 1 for all $\theta \in \Omega$. From [9] this is equivalent to say that consensus is achieved for all $\theta \in \Omega$.

(Necessity) Suppose that (1) achieves robust consensus. From [9] this implies that $D(\theta)$ has exactly one simple eigenvalue 1 and all the other eigenvalues with magnitude smaller than 1 for all $\theta \in \Omega$. This means that $D_{1}(\theta)$ is Schur for all $\theta \in \Omega$, and hence that the Lyapunov equation

$$
P(\theta)-D_{1}(\theta)^{\prime} P(\theta) D_{1}(\theta)=Q(\theta)
$$

has a unique solution $P(\theta)$ satisfying $P(\theta)>0$ for all $\theta \in \Omega$ whenever $Q(\theta)>0$ for all $\theta \in \Omega$. Since $\zeta(\theta) \neq 0$ for all $\theta \in \Omega$, this equation can be rewritten as

$$
\zeta(\theta)^{2} P(\theta)-F_{1}(\theta)^{\prime} P(\theta) F_{1}(\theta)=\zeta(\theta)^{2} Q(\theta)
$$


Let us gather the $n(n-1) / 2$ free entries of $P(\theta)$ and $Q(\theta)$ into the vectors $p(\theta)$ and $q(\theta)$. We can rewrite the previous equation as

$$
E(\theta) p(\theta)=\zeta(\theta)^{2} q(\theta) .
$$

Since the solution $P(\theta)$ exists and is unique, it follows that $E(\theta)$ is invertible for all $\theta \in \Omega$, and hence

$$
p(\theta)=\frac{\operatorname{adj}(E(\theta))}{\operatorname{det}(E(\theta))} \zeta(\theta)^{2} q(\theta) .
$$

Since the degrees of $\zeta(\theta)$ and $F_{1}(\theta)$ are not greater than $n \tau$, it follows that the degree of $E(\theta)$ is not greater than $2 n \tau$, and hence the degree of $\operatorname{adj}(E(\theta))$ is not greater than

$$
\left(\frac{1}{2}(n-1) n-1\right) 2 n \tau=\mu_{1}
$$

Let us choose $Q(\theta)=\zeta(\theta)^{-2} I$, and let us redefine $P(\theta)$ as $(-1)^{a} \operatorname{det}(E(\theta)) P(\theta)$ where $a$ is 0 if $\operatorname{det}(E(\theta))>0$ for all $\theta \in \Omega$ or 1 otherwise. It follows that $P(\theta)$ is a matrix polynomial of degree not greater than $\mu_{1}$ that satisfies the Lyapunov equation

$$
\zeta(\theta)^{2} P(\theta)-F_{1}(\theta)^{\prime} P(\theta) F_{1}(\theta)=\operatorname{det}(E(\theta)) I
$$

and, hence, (27).

Theorem 1 provides a sufficient and necessary condition for robust consensus of (1) based on the existence of a Lyapunov function polynomially dependent on the uncertainty. The degree required for achieving necessity, $\mu_{1}$, depends on the degree of the uncertain weighted adjacency matrix $G(\theta)$ and on the number of agents $n$.

The condition of Theorem 1 can be investigated through convex optimization. Specifically, let $P(\theta)$ be as in Theorem 1 , and let $H_{i}(\theta)$ and $J_{i}(\theta), i=1, \ldots, h$, be auxiliary symmetric matrix polynomials of size $(n-1) \times(n-1)$, and define

$$
\begin{aligned}
& R(\theta)=P(\theta)-\sum_{i=1}^{h} H_{i}(\theta) s_{i}(\theta) \\
& T(\theta)=\zeta(\theta)^{2} P(\theta)-F_{1}(\theta)^{\prime} P(\theta) F_{1}(\theta)-\sum_{i=1}^{h} J_{i}(\theta) s_{i}(\theta) .
\end{aligned}
$$

The following result provides a sufficient condition for establishing whether (1) achieves robust consensus based on LMIs.

\section{Corollary 1}

The condition (27) holds for some symmetric matrix polynomial $P(\theta)$ of degree $d$ if $c^{*}>0$, where 
$c^{*}$ is the solution of the following optimization problem:

$$
\begin{aligned}
& c^{*}=\sup _{c, H_{i}, J_{i}, P} c \\
& \text { s.t. }\left\{\begin{array}{l}
H_{i}(\theta) \text { is } \mathrm{SOS} \\
J_{i}(\theta) \text { is } \mathrm{SOS} \\
R(\theta)-I_{n-1} \text { is SOS } \\
T(\theta)-c I_{n-1} \text { is SOS. }
\end{array}\right.
\end{aligned}
$$

Proof Suppose that the constraints in (40) are satisfied. This implies that

$$
\left\{\begin{array}{l}
H_{i}(\theta) \geq 0 \\
J_{i}(\theta) \geq 0 \\
R(\theta)-I_{n-1} \geq 0 \\
T(\theta)-c I_{n-1} \geq 0
\end{array}\right.
$$

for all $\theta \in \mathbb{R}^{r}$. Let us consider any $\theta$ in the set $\Omega$. Since $s_{i}(\theta) \geq 0$ and $H_{i}(\theta) \geq 0$, from the third inequality we get

$$
\begin{aligned}
0 & \leq R(\theta)-I_{n-1} \\
& =P(\theta)-\sum_{i=1}^{h} H_{i}(\theta) s_{i}(\theta)-I_{n-1} \\
& \leq P(\theta)-I_{n-1}
\end{aligned}
$$

which implies that

$$
P(\theta) \geq I_{n-1} \quad \forall \theta \in \Omega \text {. }
$$

Similarly, from the inequality $T(\theta)-c I_{n-1} \geq 0$ we get

$$
\zeta(\theta)^{2} P(\theta)-F_{1}(\theta)^{\prime} P(\theta) F_{1}(\theta) \geq c I_{n-1} \quad \forall \theta \in \Omega .
$$

Therefore, if $c>0$, one has that (27) is satisfied, and hence the theorem holds.

Corollary 1 shows how the condition of Theorem 1 can be investigated through convex programming by exploiting SOS polynomials. Indeed, since establishing whether a matrix polynomial is SOS can be done through an LMI as explained in Section 2.2, it follows that the condition of Corollary 1 amounts to solving an LMI feasibility test. Let us observe that the conservatism of the condition of Corollary 1 depends on the degrees of $P(\theta)$ and of the multipliers $H_{i}(\theta)$ and $J_{i}(\theta)$.

\subsection{Single Integrator and Nonnegative Weighted Adjacency Matrices}

Let us introduce the following preliminary result, which directly extends to the case of uncertain multi-agent systems the condition given in [19] (Corollary 2.18, Lemma 2.19 and Theorem 2.20) for the case of multi-agent systems without uncertainty. 


\section{Lemma 1}

Suppose that $G(\theta)$ is nonnegative. The following three statements are equivalent.

a) The system (1) achieves robust consensus.

b) for all $\theta \in \Omega, D(\theta)$ has exactly one simple eigenvalue 1 and all the other eigenvalues satisfy $|\lambda|<1$.

c) for all $\theta \in \Omega$, the directed graph $\mathscr{G}(\theta)$ has a spanning tree.

The following result shows how Lemma 1 can be exploited to obtain a necessary and sufficient condition for robust consensus with single integrator and nonnegative weighted adjacency matrices based on the zeros of a polynomial.

\section{Theorem 2}

Suppose that $G(\theta)$ is nonnegative. The system (1) achieves robust consensus if and only if

$$
q_{D}(\theta) \neq 0 \quad \forall \theta \in \Omega
$$

where

$$
q_{D}(\theta)=\left.\frac{d l_{D}(\lambda, \theta)}{d \lambda}\right|_{\lambda=1}
$$

and

$$
l_{D}(\lambda, \theta)=\operatorname{det}(\lambda I-D(\theta)) .
$$

Proof Suppose that $G(\theta)$ is nonnegative. From Lemma 1 one has that (1) achieves robust consensus if and only if, for all $\theta \in \Omega, D(\theta)$ has exactly one simple eigenvalue 1 and all the other eigenvalues with magnitude smaller than 1 .

Now, since $D(\theta)$ is a stochastic matrix with positive diagonal elements, it follows that every eigenvalue of $D(\theta)$ not equal to 1 has magnitude smaller than 1, see e.g. [16]. Hence, it just remains to show that the eigenvalue 1 is simple.

Let us observe that this is equivalent to say that the characteristic polynomial $l_{D}(\lambda, \theta)$ of $D(\theta)$ can be written as

$$
l_{D}(\lambda, \theta)=(\lambda-1) \xi(\lambda, \theta)
$$

where

$$
\xi(1, \theta) \neq 0 \quad \forall \theta \in \Omega .
$$

This last condition coincides with (45) since

$$
\frac{d l_{D}(\lambda, \theta)}{d \lambda}=\xi(\lambda, \theta)+(\lambda-1) \frac{d \xi(\lambda, \theta)}{d \lambda}
$$

and, hence, the theorem holds. 
Theorem 2 provides a necessary and sufficient condition for robust consensus that can be used in the case of single integrator and nonnegative weighted adjacency matrices. This condition requires to check whether the polynomial $q_{D}(\theta)$ is nonzero over $\Omega$.

The condition of Theorem 2 can be investigated through convex optimization. Specifically, let us define

$$
q_{F}(\theta)=\left.\frac{d l_{F}(\lambda, \theta)}{d \lambda}\right|_{\lambda=1}
$$

where

$$
l_{F}(\lambda, \theta)=\operatorname{det}(\lambda I-F(\theta)) .
$$

Also, let $a_{i}(\theta), i=1, \ldots, h$, be auxiliary polynomials, and define

$$
b(\theta)=q_{F}\left(\theta_{0}\right) q_{F}(\theta)-\sum_{i=1}^{h} a_{i}(\theta) s_{i}(\theta)
$$

where $\theta_{0}$ is arbitrary in $\Omega$.

\section{Corollary 2}

Suppose that $G(\theta)$ is nonnegative. The condition (45) holds if $c^{*}>0$, where $c^{*}$ is the solution of the following optimization problem:

$$
\begin{aligned}
c^{*}=\sup _{a_{i}, c} c \\
\text { s.t. }\left\{\begin{array}{l}
a_{i}(\theta) \text { is } \operatorname{SOS} \\
b(\theta)-c \text { is } \operatorname{SOS} .
\end{array}\right.
\end{aligned}
$$

Proof Suppose that the constraints in (54) are satisfied. This implies that

$$
\left\{\begin{array}{l}
a_{i}(\theta) \geq 0 \\
b(\theta)-c \geq 0
\end{array}\right.
$$

for all $\theta \in \mathbb{R}^{r}$. Let us consider any $\theta$ in the set $\Omega$. Since $s_{i}(\theta) \geq 0$ and $a_{i}(\theta) \geq 0$, from the second inequality we get

$$
\begin{aligned}
0 & \leq b(\theta)-c \\
& =q_{F}\left(\theta_{0}\right) q_{F}(\theta)-\sum_{i=1}^{h} a_{i}(\theta) s_{i}(\theta)-c \\
& \leq q_{F}\left(\theta_{0}\right) q_{F}(\theta)-c
\end{aligned}
$$

which implies that

$$
q_{F}\left(\theta_{0}\right) q_{F}(\theta) \geq c \forall \theta \in \Omega
$$

If $c>0$, this implies that $q_{F}\left(\theta_{0}\right) q_{F}(\theta)$ is positive over $\Omega$. From the continuity of $q_{F}(\theta)$ and the fact that $\theta_{0} \in \Omega$, it follows that

$$
q_{F}(\theta) \neq 0 \quad \forall \theta \in \Omega
$$

By observing that

$$
q_{F}(\theta)=\zeta(\theta)^{n} D(\theta)
$$


and $\zeta(\theta) \neq 0$ for all $\theta \in \Omega$, we conclude that (45) holds.

\subsection{Double Integrators}

From (8) one has that

$$
\begin{aligned}
u_{1}(k)-u_{i}(k)= & k_{1}\left(-L_{i i}(\theta)\left(x_{1}(k)-x_{i}(k)\right)+\sum_{j=2}^{n}\left(G_{i j}(\theta)-G_{1 j}(\theta)\right)\left(x_{1}(k)-x_{j}(k)\right)\right) \\
& +k_{2}\left(-L_{i i}(\theta)\left(\varrho_{1}(k)-\varrho_{i}(k)\right)+\sum_{j=2}^{n}\left(G_{i j}(\theta)-G_{1 j}(\theta)\right)\left(\varrho_{1}(k)-\varrho_{j}(k)\right)\right) .
\end{aligned}
$$

This implies that

$$
\left\{\begin{aligned}
x_{1}(k+1)-x_{i}(k+1)= & x_{1}(k)-x_{i}(k)+\varrho_{1}(k)-\varrho_{i}(k) \\
\varrho_{1}(k+1)-\varrho_{i}(k+1)= & \varrho_{1}(k)-\varrho_{i}(k)-k_{1}\left(\sum_{j=2}^{n}\left(L_{i j}(\theta)-L_{1 j}(\theta)\right)\left(x_{1}(k)-x_{j}(k)\right)\right) \\
& -k_{2}\left(\sum_{j=2}^{n}\left(L_{i j}(\theta)-L_{1 j}(\theta)\right)\left(\varrho_{1}(k)-\varrho_{j}(k)\right)\right) .
\end{aligned}\right.
$$

Hence, (9) can be rewritten as

$$
z(k+1)=\tilde{\Gamma}(\theta) z(k)
$$

where

$$
\begin{aligned}
z(k) & =\left(x_{1}-x_{2}, \ldots, x_{1}-x_{n}, \varrho_{1}-\varrho_{2}, \ldots, \varrho_{1}-\varrho_{n}\right)^{\prime} \\
\tilde{\Gamma}(\theta) & =\left(\begin{array}{cc}
I_{n-1} & I_{n-1} \\
-k_{1} \tilde{L}(\theta) & I_{n-1}-k_{2} \tilde{L}(\theta)
\end{array}\right) \\
\tilde{L}(\theta) & =\left(\begin{array}{ccc}
L_{22}(\theta)-L_{12}(\theta) & \ldots & L_{2 n}(\theta)-L_{1 n}(\theta) \\
\vdots & \ddots & \vdots \\
L_{n 2}(\theta)-L_{12}(\theta) & \ldots & L_{n n}(\theta)-L_{1 n}(\theta)
\end{array}\right) .
\end{aligned}
$$

The following preliminary result directly extends to the case of uncertain multi-agent systems the condition given in [20] for the case of multi-agent systems without uncertainty.

\section{Lemma 2}

The system (7) achieves robust consensus if and only if

$$
\left|\lambda_{i}(\tilde{\Gamma}(\theta))\right|<1 \quad \forall i=1, \ldots, 2 n-2 \forall \theta \in \Omega
$$

where $\lambda_{i}(\tilde{\Gamma}(\theta))$ is the $i$-th eigenvalue of $\tilde{\Gamma}(\theta)$.

The following result provides a sufficient and necessary condition for establishing whether (7) achieves robust consensus. 


\section{Theorem 3}

Let $\tau$ be the degree of $G(\theta)$, and define

$$
\mu_{2}=2 n(2 n-3) \tau
$$

The system (7) achieves robust consensus if and only if there exists a symmetric matrix polynomial $P(\theta) \in \mathbb{R}^{(2 n-2) \times(2 n-2)}$ of degree $d \leq \mu_{2}$ such that

$$
\left\{\begin{array}{l}
P(\theta)>0 \\
P(\theta)-\tilde{\Gamma}(\theta)^{\prime} P(\theta) \tilde{\Gamma}(\theta)>0
\end{array} \quad \forall \theta \in \Omega .\right.
$$

Proof (Sufficiency) Suppose that (66) holds. From Lyapunov stability theorem for discrete-time linear systems, this implies that (64) holds. Hence, from Lemma 2, we conclude that (7) achieves robust consensus.

(Necessity) Suppose that (7) achieves robust consensus. From Lemma 2 this implies that (64) holds. This means that the Lyapunov equation

$$
P(\theta)-\tilde{\Gamma}(\theta)^{\prime} P(\theta) \tilde{\Gamma}(\theta)=Q(\theta)
$$

has a unique solution $P(\theta)$ satisfying $P(\theta)>0$ for all $\theta \in \Omega$ whenever $Q(\theta)>0$ for all $\theta \in \Omega$. This equation can be rewritten as

$$
E(\theta) p(\theta)=q(\theta)
$$

where $p(\theta)$ and $q(\theta)$ contain the $(2 n-1)(2 n-2) / 2$ free entries of $P(\theta)$ and $Q(\theta)$. Since the solution $P(\theta)$ exists and is unique, it follows that $E(\theta)$ is invertible for all $\theta \in \Omega$, and hence

$$
p(\theta)=\frac{\operatorname{adj}(E(\theta))}{\operatorname{det}(E(\theta))} q(\theta)
$$

Since the degree of $\tilde{\Gamma}(\theta)$ is not greater than $\tau$, it follows that the degree of $E(\theta)$ is not greater than $2 \tau$, and hence the degree of $\operatorname{adj}(E(\theta))$ is not greater than

$$
\left(\frac{1}{2}(2 n-1)(2 n-2)-1\right) 2 \tau=\mu_{2} .
$$

Let us choose $Q(\theta)=I_{2 n-2}$, and let us redefine $P(\theta)$ as $(-1)^{a} \operatorname{det}(E(\theta)) P(\theta)$ where $a$ is 0 if $\operatorname{det}(E(\theta))>0$ for all $\theta \in \Omega$ or 1 otherwise. It follows that $P(\theta)$ is a matrix polynomial of degree not greater than $\mu_{2}$ that satisfies (66).

Theorem 1 provides a sufficient and necessary condition for robust consensus of (7) based on the existence of a Lyapunov function polynomially dependent on the uncertainty. This condition can be investigated through convex optimization. 
Specifically, let $P(\theta)$ be as in Theorem 1 , and let $H_{i}(\theta)$ and $J_{i}(\theta), i=1, \ldots, h$, be auxiliary symmetric matrix polynomials of size $(2 n-2) \times(2 n-2)$, and define

$$
\begin{aligned}
& R(\theta)=P(\theta)-\sum_{i=1}^{h} H_{i}(\theta) s_{i}(\theta) \\
& T(\theta)=P(\theta)-\tilde{\Gamma}(\theta)(\theta)^{\prime} P(\theta) \tilde{\Gamma}(\theta)-\sum_{i=1}^{h} J_{i}(\theta) s_{i}(\theta) .
\end{aligned}
$$

The following result provides a sufficient condition for establishing whether (7) achieves robust consensus based on LMIs. The proof is analogous to that of Corollary 1.

\section{Corollary 3}

The condition (66) holds for some symmetric matrix polynomial $P(\theta)$ of degree $d$ if $c^{*}>0$, where $c^{*}$ is the solution of (40) with $R(\theta)$ and $T(\theta)$ replaced by those in (71).

\section{NUMERICAL EXAMPLES}

In this section we present two examples to illustrate the proposed conditions. The LMI problems are solved with MATLAB and the toolbox SeDuMi [21].

\subsection{Example 1}

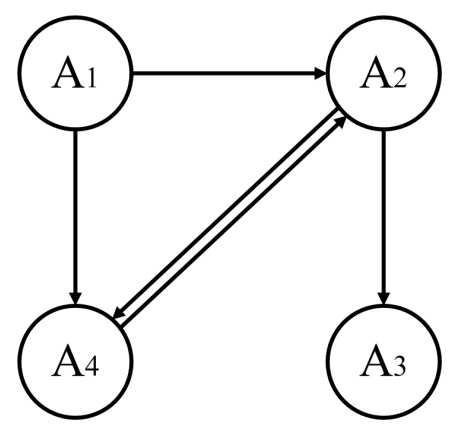

Figure 1. Topology of four-agent system.

Let us consider the four-agent system in Figure 1 with

$$
G(\theta)=\left(\begin{array}{cccc}
1 & 0 & 0 & 0 \\
1+\theta & 1 & 0 & 2 \\
0 & 1 & 1 & 0 \\
3+2 \theta & 4 & 0 & 1
\end{array}\right)
$$

where $\theta$ is constrained in

$$
\Omega=[-1,1]
$$


This set can be described as in (3) with

$$
s_{1}(\theta)=1-\theta^{2}
$$

By using (5) we get

$$
D(\theta)=\left(\begin{array}{cccc}
1 & 0 & 0 & 0 \\
\frac{1+\theta}{4+\theta} & \frac{1}{4+\theta} & 0 & \frac{2}{4+\theta} \\
0 & 0.5 & 0.5 & 0 \\
\frac{3+2 \theta}{8+2 \theta} & \frac{2}{4+\theta} & 0 & \frac{1}{8+2 \theta}
\end{array}\right)
$$

and, hence,

$$
\zeta(\theta)=8+2 \theta
$$

First, let us use Corollary 1 to establish whether robust consensus with single integrator can be achieved. We solve the LMI problem (40) with a constant symmetric matrix function $P(\theta)$ finding $c^{*}=+\infty$. Hence, from Corollary 1 we conclude that robust consensus with single integrator can be achieved.

The same conclusion can be obtained using Corollary 2 since $G(\theta)$ is nonnegative. Specifically, the polynomial $q_{F}(\theta)$ in (51) is given by

$$
q_{F}(\theta)=8 \theta^{4}+116 \theta^{3}+596 \theta^{2}+1248 \theta+832 .
$$

We solve the LMI problem (54) with a multiplier $a_{1}(\theta)$ of degree 2 , finding $c^{*}=72$. Hence, from Corollary 2 we conclude that robust consensus with single integrator can be achieved.

Second, let us use Corollary 3 to establish whether robust consensus with double integrators can be achieved. In particular, we consider (7) with $k_{1}=0.021$ and $k_{2}=0.197$. We solve the LMI problem (40) with a constant symmetric matrix function $P(\theta)$ finding $c^{*}=+\infty$. Hence, from Corollary 3 we conclude that robust consensus with double integrators can be achieved.

\subsection{Example 2}

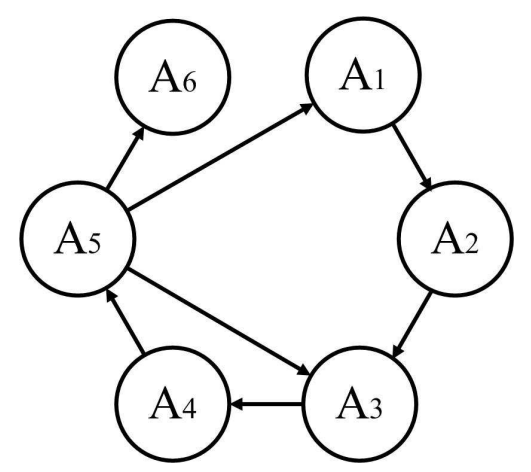

Figure 2. Topology of six-agent system. 
Let us consider the six-agent system in Figure 2 with

$$
G(\theta)=\left(\begin{array}{cccccc}
1 & 0 & 0 & 0 & 1 & 0 \\
3+\theta_{1} & 1 & 0 & 0 & 0 & 0 \\
0 & 3-\theta_{1} & 1 & 0 & \theta_{1}+\theta_{2} & 0 \\
0 & 0 & 3+\theta_{1} & 1 & 0 & 0 \\
0 & 0 & 0 & 1+0.5 \theta_{2} & 1 & 0 \\
0 & 0 & 0 & 0 & 1 & 1
\end{array}\right)
$$

where $\theta \in \mathbb{R}^{2}$ is constrained in

$$
\Omega=\left\{\theta \in \mathbb{R}^{2}:\|\theta\| \leq 1\right\} .
$$

This set can be described as in (3) with

$$
s_{1}(\theta)=1-\theta_{1}^{2}-\theta_{2}^{2}
$$

Hence, different from the Example 1, the network is affected by two uncertain parameters, and $G(\theta)$ is not nonnegative.

First, let us use Corollary 1 to establish whether robust consensus with single integrator can be achieved. We solve the LMI problem (40) with a constant symmetric matrix function $P(\theta)$ finding $c^{*}=+\infty$. Hence, from Corollary 1 we conclude that robust consensus with single integrator can be achieved. This is confirmed by Figure 3, which shows in Figure 3a a trajectory of $x(k)$ for randomly chosen $\theta \in \Omega$ and $x(0)$, and in Figure $3 \mathrm{~b} 100$ trajectories of $y(k)$, where $y_{i}(k)=x_{i}(k)-x_{1}(k)$, $i=2, \ldots, 6$, for randomly chosen $\theta \in \Omega$ and $x(0)$.

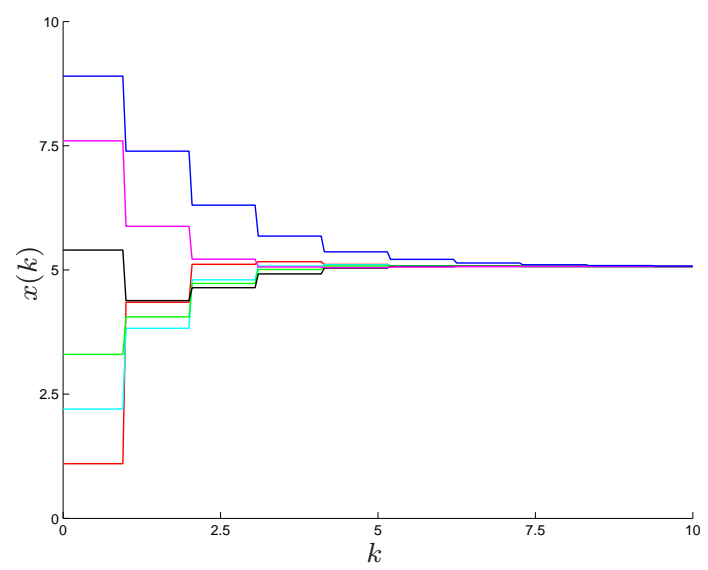

(a)

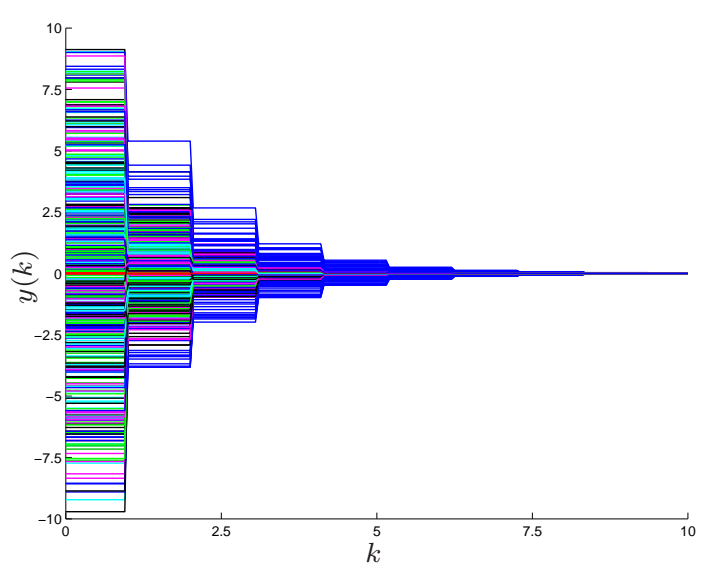

(b)

Figure 3. Example 2: some trajectories with single integrator.

Second, let us use Corollary 3 to establish whether robust consensus with double integrators can be achieved. In particular, we consider (7) with $k_{1}=0.01$ and $k_{2}=0.2$. We solve the LMI problem (40) with a symmetric matrix function $P(\theta)$ of degree 1 , finding $c^{*}=+\infty$. Hence, from Corollary 3 we conclude that robust consensus with double integrators can be achieved. This is confirmed by 
Figure 4, which shows in Figures $4 \mathrm{a}-4 \mathrm{~b}$ a trajectory of $x(k)$ and $\varrho(k)$ for randomly chosen $\theta \in \Omega$ and $x(0), \varrho(0)$, and in Figures $4 \mathrm{c}-4 \mathrm{~d} 100$ trajectories of $y(k)$ and $z(k)$, where $y_{i}(k)=x_{i}(k)-x_{1}(k)$ and $z_{i}(k)=\varrho_{i}(k)-\varrho_{1}(k), i=2, \ldots, 6$, for randomly chosen $\theta \in \Omega$ and $x(0), \varrho(0)$.

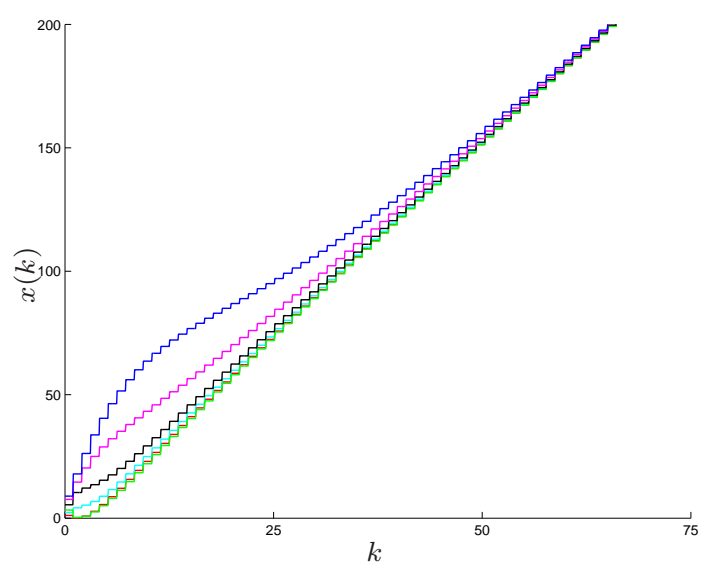

(a)

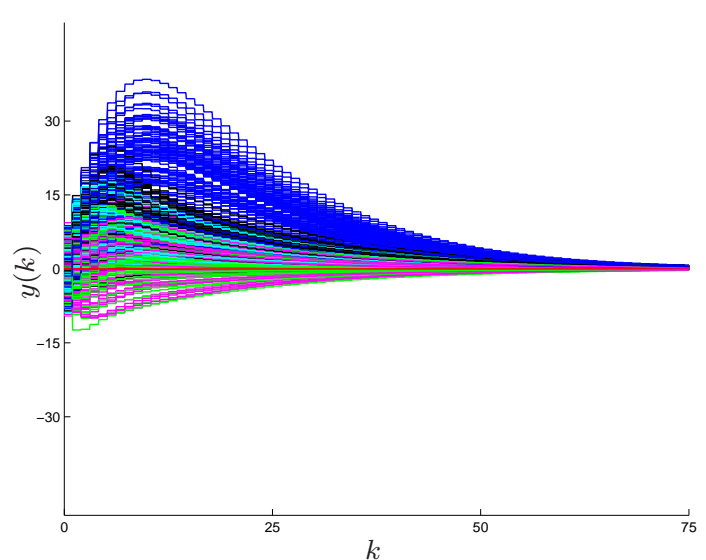

(c)

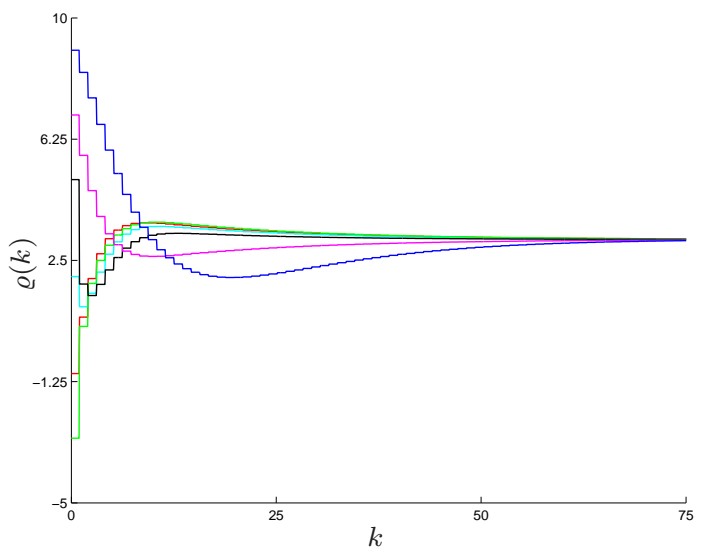

(b)

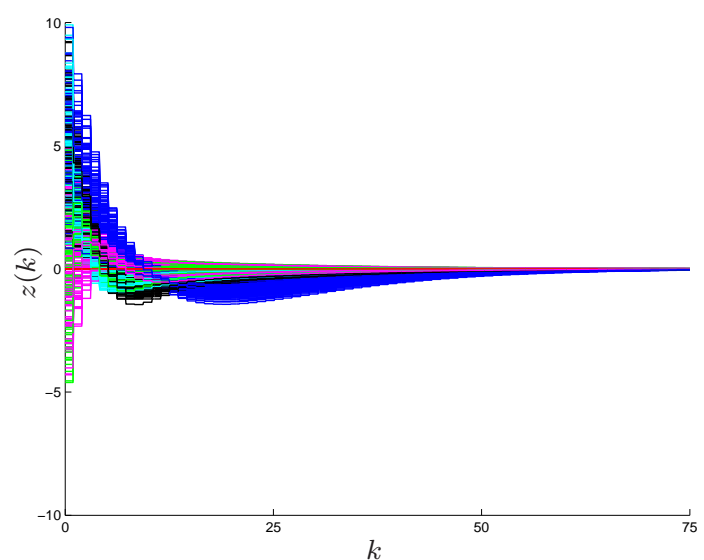

(d)

Figure 4. Example 2: some trajectories with double integrators.

\section{CONCLUSIONS}

We have investigated multi-agent systems with discrete-time dynamics where the weighted adjacency matrix is a polynomial function of uncertain parameters constrained into a semialgebraic set. We have provided necessary and sufficient conditions for robust consensus with single and double integrators based on the existence of a Lyapunov function polynomially dependent on the uncertainty. Moreover, we have provided a necessary and sufficient condition for robust consensus with single integrator and positive weighted adjacency matrices in terms of positivity of a polynomial. Lastly, we have shown how these conditions can be investigated through convex programming by exploiting LMIs and SOS polynomials. The proposed conditions can be extended 
in a number of directions, for instance to the case that the underlying systems are controlled in networked environments by adopting the frameworks introduced in [22-24].

\section{REFERENCES}

1. Gao Y, Wang L. A necessary and sufficient condition for consensus of continuous-time agents over undirected time-varying networks. IEEE Transactions on Automatic Control 2011; 56(5):1226-1231.

2. Tian Y, Liu C. Consensus of multi-agent systems with diverse input and communication delays. IEEE Transactions on Automatic Control 2008; 53(9):2122-2128.

3. Nuño E, Ortega R, Basañez L, Hill D. Synchronization of networks of nonidentical euler-lagrange sytems with uncertain parameters and communication delays. IEEE Transactions on Automatic Control 2011; 56(4):935-941.

4. Gao Y, Wang L. Sampled-data based consensus of continuous-time multi-agent systems with time-varying topology. IEEE Transactions on Automatic Control 2011; 56(5):1226-1231.

5. You K, Xie L. Network topology and communication data rate for consensusability of discrete-time multi-agent systems. IEEE Transactions on Automatic Control 2011; 56(10):2262-2275.

6. Cheng L, Hou Z, Tan M, Wang X. Necessary and sufficient conditions for consensus of double integrator muti-agent systems with measurement noises. IEEE Transactions on Automatic Control 2011; 56(8):1958-1963.

7. Yu W, Chen G, Cao M. Some necessary and sufficient conditions for second-order consensus in multi-agent dynamical systems. Automatica 2010; 46(6):1089-1095.

8. Li T, Zhang J. Consensus conditions of multi-agent systems with time-varying topologies and stochastic communication noises. IEEE Transactions on Automatic Control 2010; 55(9):2043-2057.

9. Ren W, Beard R. Consensus seeking in multiagent systems under dynamically changing interaction topologies. IEEE Transactions on Automatic Control 2005; 50(5):655-661.

10. Abaid N, Porfiri M. Consensus over numerosity-constrained random network. IEEE Transactions on Automatic Control 2011; 56(3):649-654.

11. Tian Y, Liu C. Robust consensus of multi-agent systems with diverse input delays and asymmetric interconnection perturbations. Automatica 2009; 45:1347-1353.

12. Zhang W, Zheng Z, Guo Y. Robust consensus for uncertain multi-agent systems on directed communication topologies. in Proceedings of IEEE Conference on Decision and Control, Atlanta, GA, 2010; 6317-6322.

13. Münz U, Papachristodoulou A, Allgöwer F. Robust consensus controller design for nonlinear relative degree two multi-agent systems with communication constraints. IEEE Transactions on Automatic Control 2011; 56(1):145151.

14. Chesi G. LMI techniques for optimization over polynomials in control: a survey. IEEE Transactions on Automatic Control 2010; 55(11):2500-2510.

15. Han D, Chesi G, Hung YS. Synchronization seeking in multi-agent dynamic systems with communication uncertainties. 2011 IEEE International Symposium on Computer-Aided Control System Design (CACSD), Denver, CO, 2011; 656-661.

16. Horn R, Johnson C. Matrix Analysis. Cambridge University Press, 1985.

17. Chesi G, Garulli A, Tesi A, Vicino A. Solving quadratic distance problems: an LMI-based approach. IEEE Transactions on Automatic Control 2003; 48(2):200-212.

18. Chesi G, Garulli A, Tesi A, Vicino A. Polynomially parameter-dependent Lyapunov functions for robust stability of polytopic systems: an LMI approach. IEEE Transactions on Automatic Control 2005; 50(3):365-370.

19. Ren W, Beard R. Distributed Consensus in Multi-vehicle Cooperative Control. Communications and Control Engineering, Springer-Verlag, 2008.

20. Zhang Y, Tian Y. Consentability and protocol design of multi-agent systems with stochastic switching topology. Automatica 2009; 45:1195-1201.

21. Sturm J. Using SeDuMi 1.02, a MATLAB toolbox for optimization over symmetric cones. optimization Methods and Software 1999; 11-12:625-653.

22. Zhang L, Gao H, Kaynak O. Survey of studies on network-induced constraints in networked control systems. IEEE Transactions on Industrial Informatics in press; .

23. Zhang L, Lam J. Necessary and sufficient conditions for analysis and synthesis of Markov jump linear systems with incomplete transition descriptions. IEEE Transactions on Automatic Control 2010; 55(7):1695-1701. 
24. Lu Q, Zhang L, Basin M, Tian H. Analysis and synthesis for networked control systems with uncertain rate of packet losses. Journal of the Franklin Institute 2012; 349(5):2500-2514. 